\title{
The use of PBPK/PD to establish clinically relevant dissolution specifications for zolpidem immediate release tablets
}

\author{
Rafael L.M. Paraiso ${ }^{a}$, Rachel H. Rose ${ }^{c}$, Nikoletta Fotaki ${ }^{\mathrm{d}}$, Mark McAllister ${ }^{\mathrm{e}}$, \\ Jennifer B. Dressman ${ }^{\mathrm{a}, \mathrm{b}, *}$ \\ a Institute of Pharmaceutical Technology, Goethe University, Germany \\ ${ }^{\mathrm{b}}$ Fraunhofer Institute for Molecular Biology and Applied Ecology IME, Division of Translational Medicine and Pharmacology, Max von Lauer St. 9, Frankfurt am Main, \\ 60438, Germany \\ ${ }^{\mathrm{c}}$ Certara UK Limited, Simcyp Division, Level-2-Acero, 1 Concourse Way, Sheffield, S1 2NJ, United Kingdom \\ ${ }^{\mathrm{d}}$ Department of Pharmacy and Pharmacology, Faculty of Science, University of Bath, Bath, United Kingdom \\ ${ }^{\mathrm{e}}$ Pfizer Drug Product Design, Sandwich, UK
}

\section{A R T I C L E I N F O}

\section{Keywords:}

PBPK

$\mathrm{PBPK} / \mathrm{PD}$

Zolpidem

Clinical relevant dissolution specification

Food effect

\begin{abstract}
A B S T R A C T
Background: Zolpidem is a non-benzodiazepine hypnotic agent which has been shown to be effective in inducing and maintaining sleep in adults and is one of the most frequently prescribed hypnotics in the world. For drugs that are used to treat sleeping disorders, the time to reach the maximum concentration $\left(\mathrm{T}_{\max }\right)$ of the drug in plasma is important to achieving a fast onset of action and this must be maintained when switching from one product to another.

Objectives: The main objective of the present work was to create a PBPK/PD model for zolpidem and establish a clinically relevant "safe space" for dissolution of zolpidem from the commercial immediate release (IR) formulation. A second objective was to analyze literature pharmacokinetic data to verify the negative food effect ascribed to zolpidem and consider its ramifications in terms of the "safe space" for dissolution.

Methods: Using dissolution, pharmacokinetic and pharmacodynamic data, an integrated PBPK/PD model for immediate release zolpidem tablets was constructed in Simcyp ${ }^{\circledast}$. This model was used to identify the clinically relevant dissolution specifications necessary to ensure efficacy.

Results: According to the simulations, as long as $85 \%$ of the drug is released in 45 minutes or less, the impact on the PK and PD profiles of zolpidem would be minimal. According to the FDA, the drug has to dissolve from the test and reference products at a similar rate and to an extent of $85 \%$ in not more than 30 minutes to pass bioequivalence via the BCS-biowaiver test. Thus, the BCS-biowaiver specifications are somewhat more stringent than the "safe space" based on the PBPK/PD model. Published data from fasted and fed state pharmacokinetic studies suggest but do not prove a negative food effect of zolpidem.

Conclusions: A PBPK/PD model indicates that current BCS-biowaiver criteria are more restrictive for immediate release zolpidem tablets than they need to be. In view of the close relationship between PK and PD, it remains advisable to avoid taking zolpidem tablets with or immediately after a meal, as indicated by the Stilnox ${ }^{\circledR}$ labeling.
\end{abstract}

\section{Introduction}

Zolpidem is a non-benzodiazepine hypnotic agent which has been shown to be effective in inducing and maintaining sleep in adults and is one of the most frequently prescribed hypnotics in the world (Norman et al., 2017; Salvà and Costa, 1995). Factors contributing to its success are the rapid absorption of zolpidem after oral ingestion, which facilitates rapid induction of sleep, and the short elimination half-life (2.4h), which leads to fewer residual ("hangover") effects compared with other options used for sleep induction (de Haas et al., 2010; Vermeeren, 2004).

The mechanism of action of this imidazopyridine comprises interactions at the benzodiazepine $\omega 1$-receptor subtype, with its hypnoticsedative action exclusively attributable to agonist binding to the $\alpha 1$ gamma-aminobutyric acid type $\mathrm{A}\left(\mathrm{GABA}_{\mathrm{A}}\right)$ receptors. The $\alpha 1-\mathrm{GABA}$ receptor is essentially associated with sedation, whereas other receptor

\footnotetext{
* Corresponding author at:

E-mail address: dressman@em.uni-frankfurt.de (J.B. Dressman).
} 
subtypes are responsible for the various further effects of GABAergic ligands, such as muscle relaxation, anxiolytics and amnesia (de Haas et al., 2010). The selectivity of zolpidem's binding explains why it has a strong sedative effect, but only minor activity as an anxiolytic, myorelaxant and anticonvulsant (Salvà and Costa, 1995).

For drugs that are used as hypnotics to treat sleeping disorders, the time to reach the maximum concentration $\left(\mathrm{T}_{\max }\right)$ of the drug in plasma is important to achieving a fast onset of action (Greenblatt, 2010). As food intake can delay the gastric emptying time and thus the absorption of drugs with short half-lives, such as zolpidem, the rate and potentially the extent of drug absorption can be reduced if administered in the fed state, leading to treatment failure (Eller and Della-Coletta, 1990; Greenblatt et al., 1978; Locniskar et al., 1984; Ms et al., 2007). Notably for zolpidem, the labeling of the commercial product describes zolpidem as having a negative food effect. This applies to the immediate release (IR), modified release (MR) and sublingual (SL) formulations of zolpidem (Andreas et al., 2017; FDA, 2007 ; Paraiso et al., 2019).

In general, dissolution tests on oral drug products are performed with media that are described in the pharmacopeia. These tests are often used in the development of drug products containing highly soluble drugs to guide the formulation process and in quality control testing of the commercial product (Tsume et al., 2018). For poorly soluble drugs, biorelevant dissolution media which simulate the composition of gastrointestinal environment fluids are often more appropriate (Fotaki and Vertzoni, 2010; Jantratid et al., 2009; Klein, 2010; Markopoulos et al., 2015). Biorelevant media have also been applied to set clinically relevant dissolution specifications (CRDS) and to establish a dissolution "safe space" for drug formulations. To set CRDS, the acceptance criteria for the in vitro dissolution test must be linked to the in vivo performance of drug formulation (Hermans et al., 2017; Dickinson et al., 2008; Tsume et al., 2018). However, establishing this relationship between dissolution performance and in vivo results is not always straightforward.

Recent advances in physiologically based pharmacokinetic (PBPK) modeling with different commercial platforms available in the market has facilitated the translation of in vitro dissolution data into predictions of the in vivo plasma profile of drugs to set the CRDS (Hermans et al., 2017; Tsume et al., 2018). Going one step further, it is also possible to couple PBPK platforms with pharmacodynamic (PD) models. Using this approach, there is a possibility to integrate dissolution data into the PBPK model and couple it with the PD model to establish a truly clinically driven "safe space" for the dissolution of the drug formulation (Cristofoletti and Dressman, 2014).

The main objective of the present work was to create a PBPK/PD model for zolpidem in Simcyp ${ }^{\circledR}$ and establish a safe space for the dissolution of zolpidem from the commercial immediate release (IR) formulation using dissolution, pharmacokinetic and pharmacodynamic data. According to the Biopharmaceutical Classification System (BCS) proposed by Amidon and co-workers (1995) zolpidem is a member of BCS class I, being characterized as a highly soluble and highly permeable weakly basic compound with a pKa of 6.16 (Amidon et al., 1995; Colo et al., 2015; Ramirez et al., 2010). Further, since zolpidem (IR) has been described as having a negative food effect, the impact of time of dosing on PK/PD outcomes was also explored.

\section{Material and methods}

\subsection{Materials}

The zolpidem dissolution profiles used to build the PBPK/PD model was performed with Stilnox ${ }^{\circledast}$ tablets (Batch No. \#70662; SanofiAventis, Gentilly, France). The in vitro dissolution profiles of Stilnox ${ }^{\circledast}$ tablets were obtained from Paraiso and co-workers (2019).
Table 1

Composition and physicochemical characteristics of Level II biorelevant media for simulating the environment of gastric and the small intestine after a low fat meal.

\begin{tabular}{lll}
\hline Gastrointestinal segment & Stomach & $\begin{array}{l}\text { Small Intestine } \\
\text { (proximal) } \\
\text { Level II - FeSSIF V2 }\end{array}$ \\
Biorelevant media & $\begin{array}{l}\text { Level II - } \\
\text { FeSSGF }_{\text {middle }}\end{array}$ & \\
\hline Acetic acid (mM) & 18.31 & - \\
Sodium acetate (mM) & 32.98 & - \\
Maleic acid (mM) & - & 71.9 \\
Sodium hydroxide (mM) & - & 102.4 \\
Lipofundin / buffer & $8.75: 91.25$ & - \\
Sodium taurocholate (mM) & - & 10 \\
Lecithin (mM) & - & 2 \\
Glyceryl monooleate (MmM) & - & 5 \\
Sodium oleate (mM) & - & 0.8 \\
Sodium chloride (mM) & 181.7 & 125.5 \\
Osmolality (mOsm/kg) & 400 & 390 \\
Buffer capacity (HCl) $[(\mathrm{mmol} / \mathrm{l}) /$ & 25 & 25 \\
$\quad \Delta$ pH] & 5.0 & 5.8 \\
pH & & \\
\hline
\end{tabular}

\subsection{Biorelevant dissolution tests}

Although as a BCS Class I drug, compendial media would be appropriate for studying dissolution of zolpidem in the fasted state, to simulate the food effect in vitro it is necessary to use biorelevant media (Markopoulos et al., 2015). Data for biorelevant dissolution simulating the fasted and fed states were taken from a previous publication by Paraiso and co-workers (2019) (Paraiso et al., 2019). The data were digitized using the software WebPlotDigitizer v. 4.2 (San Francisco, USA; source: https://automeris.io/WebPlotDigitizer). The composition of the biorelevant media used to simulate the fed state is summarized in Table 1. The biorelevant dissolution tests were performed using USP 2 dissolution test apparatus (Erweka DT 80, Heusenstamm, Germany) at $37 \pm 0.5^{\circ} \mathrm{C}$ in $500 \mathrm{~mL}$ medium. The dissolution behavior of Stilnox ${ }^{\circledast}$

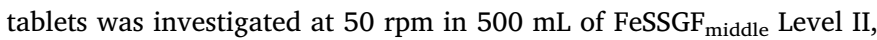
and in FeSSIF-V2 Level II (pH 5.8). FeSSGF middle (pH 5.0 and $1.75 \%$ fat) is the biorelevant media that represents the period from 75-165 minutes after food ingestion. This medium was selected because in the clinical study, Stilnox ${ }^{\circledast}$ tablets were ingested after a low fat breakfast (Greenblatt et al., 2006). Sampling for all dissolution tests was performed at $5,10,20,30,40,50,60$ and 120 minutes.

\section{3. $P B P K$ model}

The PBPK model was built using the Simcyp ${ }^{\circledR}$ Simulator (V18.1; Certara, Sheffield, UK). Zolpidem parameters inputted into the PBPK model are shown in Table 2. The required data regarding drug physicochemical and pharmacokinetic properties were obtained from the literature sources cited in the table.

To best describe the pre-absorptive processes, i.e. drug dissolution in the gastrointestinal tract, the advanced dissolution, absorption and metabolism (ADAM) module was used. The framework of the ADAM module has already been described in the literature (Cristofoletti and Dressman, 2014; Darwich et al., 2010; Jamei et al., 2009). There are different ways to input dissolution data into this module. The first possibility is to input the dissolution profiles simulating the gastric and small intestine environment directly into the platform, the second is to use a Weibull function to fit the data and the third is to insert the solubility and dissolution data into the diffusion layer model (DLM) function. In this work we used the first option, inserting the dissolution profile directly into the respective gastrointestinal segments. Thus, the dissolution profiles were entered separately into the stomach (results in FeSSGF $_{\text {middle }}$ ) and the small intestine (results in FeSSIF-V2) segments using the piecewise cubic polynomial approach as the interpolation 
Table 2

Parameters used to implement the zolpidem PBPK model (Simcyp Simulator ${ }^{\circledR}$ V18.1).

\begin{tabular}{|c|c|c|}
\hline Parameters & Value & Reference/Comments \\
\hline Molecular weight (g/mol) & 307.39 & Salvà and Costa, 1995 \\
\hline $\log \mathrm{P}$ & 2.42 & Salvà and Costa, 1995 \\
\hline Compound type & Monoprotic base & Salvà and Costa, 1995 \\
\hline $\mathrm{pKa}$ & 6.16 & Salvà and Costa, 1995 \\
\hline Absorption (low fat fed model) & ADAM model & \\
\hline Dissolution level II FeSSGF middle & Dissolution profile & Paraiso et al., 2019 \\
\hline Dissolution level II FeSSIF-V2 & Dissolution profile & Paraiso et al., 2019 \\
\hline $\mathrm{P}_{\text {eff }}$ & $6.5 \times 10^{-4} \mathrm{~cm} / \mathrm{s}$ & Andreas et al., 2017b \\
\hline Absorption (fasted model) & ADAM model & \\
\hline Dissolution level II FaSSGF & Dissolution profile & Paraiso et al., 2019 \\
\hline Dissolution level II FaSSIF-V2 & Dissolution profile & Paraiso et al., 2019 \\
\hline$P_{\text {eff }}$ & $6.5 \times 10^{-4} \mathrm{~cm} / \mathrm{s}$ & Andreas et al., 2017b \\
\hline Distribution & Minimal PBPK model & \\
\hline Main binding protein & albumin & Salvà and Costa, 1995 \\
\hline Vss $(\mathrm{L} / \mathrm{kg})$ & 0.54 & Durand et al., 1992 \\
\hline Fu gut & 0.035 & predicted using Simcyp ${ }^{\circledast}$ \\
\hline Blood to plasma coefficient (B:P) & 0.76 & Salvà and Costa, 1995 \\
\hline Fu plasma & 0.08 & Durand et al., 1992 \\
\hline \multicolumn{3}{|l|}{ Elimination } \\
\hline Oral clearance $(\mathrm{L} / \mathrm{h})$ & 16.56 & Greenblatt et al., 2006a \\
\hline CLR (renal clearance) $(\mathrm{L} / \mathrm{h})$ & 0.18 & Simcyp ${ }^{\circledast}$ default \\
\hline PD model & Power function & \\
\hline Exponent (m) & 1.17 & Estimated form Greenblatt et al., 2006a \\
\hline Slope $(\alpha)$ & 21.2 & Estimated from Greenblatt et al., 2006a \\
\hline $\mathrm{E}_{0}$ & 0 & \\
\hline Baseline type & Additive & \\
\hline \multicolumn{3}{|l|}{ Trial condition } \\
\hline Formulation option in the software & Immediate release (IR) with interpolation of the observed in vitro dissolution data. & Default setting in Simcyp ${ }^{\circledast}$ \\
\hline Population modeling & $\begin{array}{l}\text { Virtual population trials were conducted using } 700(70 \times 10) \text { male and female healthy } \\
\text { volunteers, age ranged } 18-45 \text { years for each study. }\end{array}$ & Greenblatt et al., 2006a \\
\hline
\end{tabular}

method.

Since zolpidem has a low to moderate volume of distribution $\left(\mathrm{V}_{\mathrm{ss}}\right)$ with a value of $0.54 \mathrm{~L} / \mathrm{kg}$, the minimal PBPK model was applied to predict the plasma profile of this drug. Additionally, the unbound fraction of $8 \%$ in plasma, which was reported by Durand and coworkers (1992), was applied in the model (Durand et al., 1992).

To predict the elimination phase of the drug, the oral clearance data reported by Greenblatt and co-workers (2006) were used. For healthy adults the renal clearance $\left(\mathrm{CL}_{\mathrm{R}}\right)$ was $0.18(\mathrm{~L} / \mathrm{h})$ (Greenblatt et al., 2006).

\subsubsection{Clinical study}

In the clinical studies realized by Greenblatt and co-workers (2006) zolpidem immediate release tablets were administered to men and women two hours after intake of a light breakfast (Greenblatt et al., 2006). These conditions were modeled in Simcyp ${ }^{\circledast}$ by running simulations in a "healthy volunteer" cohort with the same gender ratio and age as the study cohort, in which the gastrointestinal physiology was adapted to represent conditions after ingestion of a low fat meal.

\subsubsection{Verification of the $P B P K$ model using an internal $P K$ data set}

The parameters and conditions of the trials depicted in Table 2 were used to build the PBPK model. The validation of the PBPK/PD model for this drug was done using an internal validation approach adopting the pharmacokinetic and pharmacodynamic in vivo data from Greenblatt and co-workers (2006). It was not possible to use external data to validate the method since no other studies using similar trial conditions (men and women, light breakfast) could be found in the open literature.

\subsection{PD model}

\subsubsection{Percentage of $\beta$-EEG variation}

To evaluate the hypnotic effect of drugs, different measures are described in the literature. Some of them are considered subjective e.g. observationally rated sedation, self-rated sedation, digit symbol substitution test (DSST), while others are more objective e.g. variations in $\alpha, \beta$ and $\gamma$ brain waves (Cysneiros et al., 2007; de Haas et al., 2010; Drover et al., 2000; Greenblatt et al., 2006, 1998).

Since the $\%$ change in the $\beta$-EEG amplitude is objective and is the outcome most often used to describe the hypnotic effects of zolpidem, we used this pharmacodynamic parameter to build the PD model.

Results for \% change in $\beta$-EEG were taken from a published PK/PD study that was used to compare the kinetics and dynamics of zolpidem IR with the modified release (MR) formulation. This study was conducted with 70 male and female healthy volunteers with $46 \%$ women enrolled (Greenblatt et al., 2006). Since the time to reach the maximum drug concentration in plasma $\left(\mathrm{T}_{\max }\right)$ and the time to reach the maximum pharmacological response $\left(\mathrm{TR}_{\max }\right)$, together with maximum plasma concentration $\left(\mathrm{C}_{\max }\right)$ and the maximal response $\left(\mathrm{R}_{\max }\right)$, showed an almost linear correlation, with no hysteresis (Fig. 1), the authors concluded that the $\%$ change in the $\beta$-EEG profile after treatment with zolpidem would be well described by a single exponential equation:

$y=\alpha x^{m}$

For this reason, we used the power function model (the default model in the software) to develop the PD model in Simcyp ${ }^{\circledast}$. The power function model is represented by the following equation:

$R 1=E_{0}+\alpha \cdot(\text { PKInput })^{m}$

where $\mathrm{R} 1$ is the pharmacological response, $\mathrm{E}_{0}$ is the initial \% change in the $\beta$ EEG wave (in our model $\mathrm{E}_{0}=0$ ), $\alpha$ is a linear coefficient, PKInput is the plasma profile concentration entered for time t, and $m$ is the exponential coefficient of the equation. The in vivo data obtained from Greenblatt and co-workers (2006) were used to estimate the $\alpha$ and $\mathrm{m}$ parameters of the model (Greenblatt et al., 2006). 


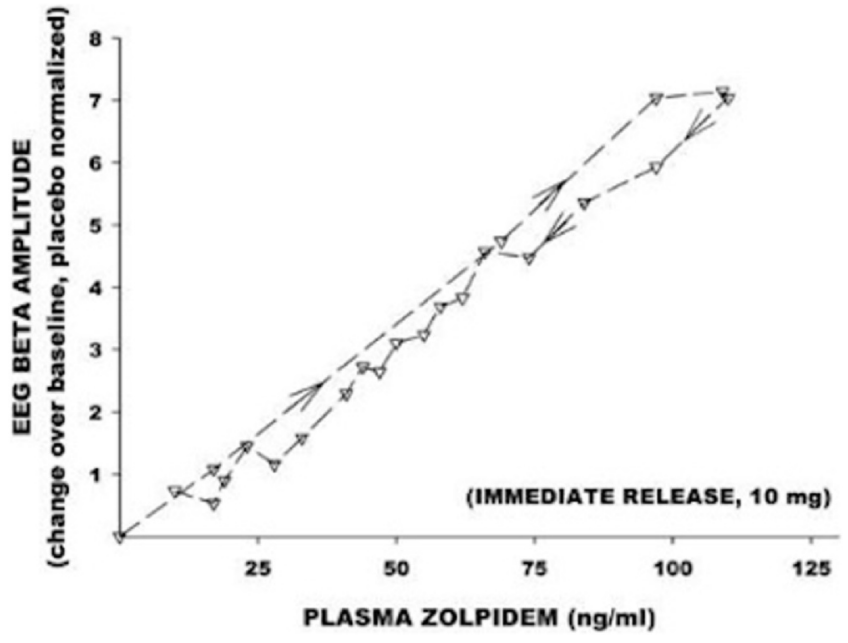

Fig. 1. Relationship between mean plasma zolpidem concentration and mean changes in the percentage electroencephalographic (EEG) of $\beta$ brain waves at corresponding times. Arrows indicate the direction of increasing time. Reproduced with permission from Greenblatt and co-workers (2006).

\section{5. $P K / P D$ simulations}

Simcyp Simulator ${ }^{\circledR}$ version 18.1 was used to predict the plasma concentration and the $\%$ change in the $\beta$ wave by zolpidem. The virtual population was selected to closely match the population enrolled by Greenblatt and co-workers (2006) with regard to age range and gender ratio (Greenblatt et al., 2006). The virtual trials simulating the "low fat" fed state were performed on the basis of administering zolpidem IR tablets with $250 \mathrm{~mL}$ of water 2 hours after a light breakfast. Starting with the intake of the drug, the clinical trial was conducted over $12 \mathrm{~h}$. The following simulations were performed:
- To evaluate the hypnotic effect of zolpidem using the \% change in the $\beta$-waves, a virtual trial was conducted enrolling 70 adults aging 19-45 years (46\% females) receiving a single oral dose of $10 \mathrm{mg}$ zolpidem administered as the IR tablet. The predicted data were compared with the observed data to internally verify the model, as no other publication with a similar trial design could be located in the open literature.

- To determine the key parameters in the PBPK/PD model, a sensitivity analysis was performed. The effect of two different input variables on the PK and PD responses of zolpidem was evaluated using the 'one-at-a-time' variation approach. Gastric emptying time (GET) (with values ranging from 0.4 to $2.0 \mathrm{~h}$ ) and effective permeability $\left(\mathrm{P}_{\text {eff }}\right.$ ) (with values ranging from 4 to $8 \times 10^{-4} \mathrm{~cm} / \mathrm{s}$ ) were the two variables tested.

- To set clinically relevant dissolution specification for zolpidem IR tablets, the model developed for zolpidem administered after a low fat meal was extrapolated to the fasted state. In order to simulate the plasma profile and the $\%$ change in $\beta$-EEG amplitude in the fasted state, the same structural model as used for the low fat fed state was applied. All parameters (Table 2) were kept constant, with the exceptions that the dissolution under conditions representing the fasted state and the default values in Simcyp ${ }^{\circledast}$ for the healthy population in the fasted state were applied. After the simulation in the fasted condition was generated, the plasma profile was verified with external data from Weinling and co-workers (2006) (Weinling et al., 2006b). This study was conducted in 24 healthy male volunteers, age range 18-45 years, under fasting conditions. The volunteers received $10 \mathrm{mg}$ zolpidem hemitartarate IR tablets in a cross-over design. . After constructing and verifying this scenario, different dissolution rates in a fasted state medium adjusted to $\mathrm{pH} 6.8$ (ranging from $85 \%$ dissolved in $15 \mathrm{~min}$ to $85 \%$ dissolved in $120 \mathrm{~min}$ ) were simulated and then entered into the model to generate the PK and PD responses of zolpidem under these conditions. These simulations were conducted in 10 virtual trials, each enrolling 70 adults aged 18-40 years and receiving a dose of $10 \mathrm{mg}$ of zolpidem IR tablets on an empty stomach.

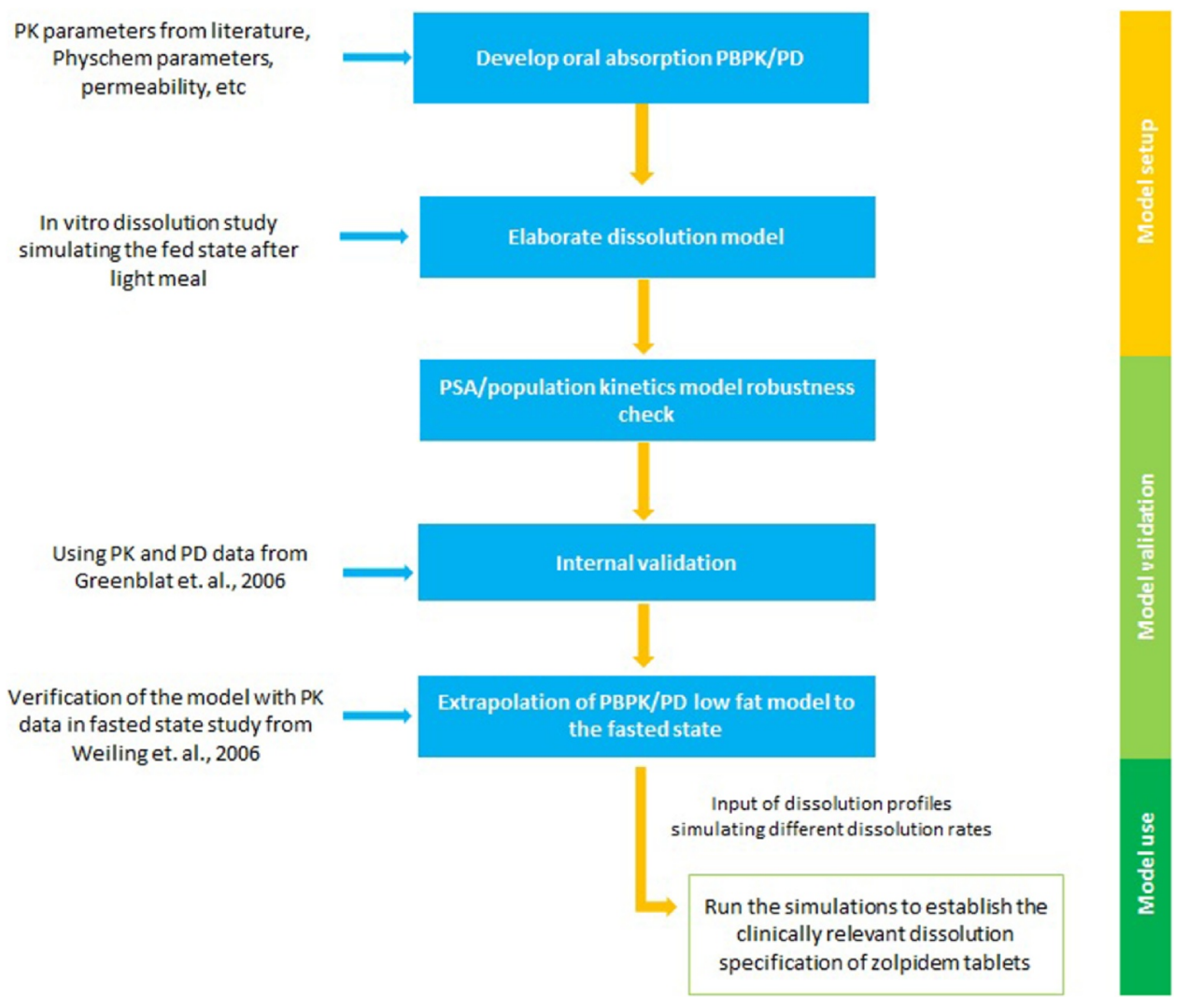

Fig. 2. PBPK/PD model strategy for zolpidem immediate release tablets 
A schematic of the steps used to create the models is shown in Fig. 2.

\subsection{Data presentation and statistical analysis}

The simulated (predicted) plasma profiles using Simcyp ${ }^{\circledR}$ software were compared with in vivo data (observed) using the average fold error (AFE) and absolute average fold error (AAFE) (Obach et al., 1997; Poulin and Theil, 2009).

$A F E=10^{\frac{1}{n} \sum \mathrm{i} \text { predicted }_{i}}$

where $\mathrm{n}$ is the numbe $\mathrm{r}$ of time points at which the concentration was ${\text { obtained, } \text { predicted }_{i} \text { and observed }}_{i}$ are the predicted and observed concentrations at a given time point $i$. The average fold error indicates whether the predicted profile underestimates or overestimates the observed values (as shown in Eq. 3): if the AFE value is less than 1 there is an underestimation and if the AFE value is greater than 1 there is an overestimation of the observed value.

The absolute average fold error quantifies the absolute error from the true value (as shown in Eq. 4). If AAFE $\leq 2$, the simulation is usually considered acceptable (Obach et al., 1997; Poulin and Theil, 2009).

$A A F E=10^{\frac{1}{n} \sum\left|\log \frac{\text { predicted }_{i}}{\text { observed }_{i}}\right|}$

\section{Results}

\subsection{Development and verification of the PBPK/PD model of zolpidem}

Fig. 3 depicts the dissolution profiles of $10 \mathrm{mg}$ Stilnox ${ }^{\circledR}$ immediate release tablets in biorelevant media simulating the gastric (FeS$\mathrm{SGF}_{\text {middle }}$ ) and upper intestinal (FeSSIF-V2) environment after ingesting a low fat meal (Paraiso et al., 2019). This figure shows that there were only small differences in the rate and extent of dissolution comparing the gastric (lower) and intestine environment (higher).

In order to simulate the "low fat" fed state plasma and pharmacodynamic profile of zolpidem, the dissolution profiles simulating the gastric and small intestine were inputted into the PBPK/PD model in Simcyp $^{\circledast}$ together with drug properties and pharmacokinetics parameters listed in Table 2.

Fig. 4 depicts the simulated and observed mean zolpidem plasma concentration and mean $\%$ change in the $\beta$-waves profiles together with the predicted $5^{\text {th }}$ and $95^{\text {th }}$ percentiles around the means.

Table 3 summarizes the calculated PK parameters $\left(\mathrm{T}_{\max }, \mathrm{C}_{\max }\right.$ and AUC), along with AFE and AAFE values. The results indicate that dissolution profiles obtained with FeSSGF middle $_{\text {and FeSSIF-V2 combined }}$

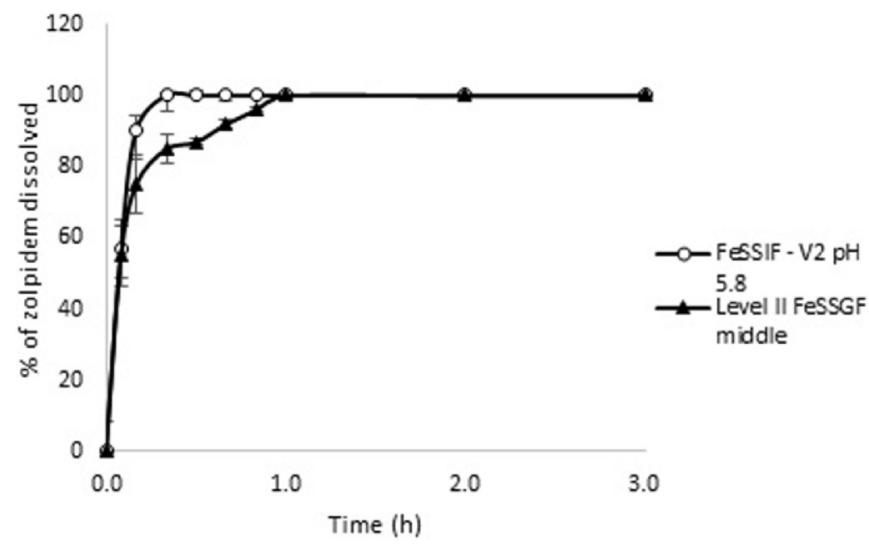

Fig. 3. Mean dissolution profiles of $10 \mathrm{mg}$ immediate release Stilnox ${ }^{\circledR}$ tablets in biorelevant Level II dissolution media simulating the low fat fed state using USP apparatus II. Reproduced with permission from Paraiso et. al (2019). with the PBPK model were able to predict the in vivo behavior of zolpidem IR tablets. The AFE for the simulation was 1.07 and the AAFE was 1.10 , indicating that the developed model predicted the plasma profile of zolpidem accurately.

Table 4 reports the simulated and observed PD parameters for zolpidem. As for the PK parameters, the simulated PD parameters $\left(R_{\max }\right.$ and AUCR and $\mathrm{TR}_{\max }$ ) were very close to those obtained in the in vivo study. The AFE was 1.11 and the AAFE was 1.13, indicating that the developed model was able to closely predict the pharmacodynamic as well as the pharmacokinetic profile of the drug.

Fig. 5 depicts the relationship between plasma concentration and pharmacodynamic response for zolpidem. The relationship between PK and $\mathrm{PD}$ has $\mathrm{r}^{2}$ of 0.9969 , indicating the excellent correlation between the plasma concentration of the drug and the PD response.

3.1.1. Parameter sensitivity analysis (PSA) to determine the effect of gastric emptying time (GET) and effective permeability $\left(P_{\text {eff }} \times 10^{-4}\right)$ on the zolpidem $P K$ profile

After developing and validating the PBPK/PD for zolpidem, a parameter sensitivity analysis (PSA) was conducted to determine which parameters could be important to establish the plasma profile and or PD response for zolpidem. Since the pharmacodynamic response is linearly related to the pharmacokinetics, the pharmacodynamic profiles are not shown.

Fig. 6a depicts how the plasma profile of zolpidem changes as a function of gastric emptying. Sensitivity analysis demonstrated that at longer gastric emptying times the in vivo $\mathrm{C}_{\max }$ and AUC were underpredicted and the $\mathrm{T}_{\max }$ was longer. The simulations came closest to the observed mean $\mathrm{C}_{\max }$ when a GET between 0.8 and $1.0 \mathrm{~h}$ was applied, and the $T_{\max }$ was best simulated by a GET of $0.9 \mathrm{~h}$. These results suggest that GET may play a role in zolpidem absorption and thus onset of action of zolpidem. By contrast, $\mathrm{P}_{\text {eff }}$ (Fig. $6 \mathrm{~b}$ ) did not change the PK profiles significantly over the range tested.

\subsection{Dissolution profile to generate the clinical relevance of dissolution for zolpidem}

Fig. 7 depicts the dissolution profile of $10 \mathrm{mg}$ Stilnox ${ }^{\circledR}$ immediate release tablets in biorelevant media simulating the gastric (FaSSGF) and upper intestinal (FaSSIF-V2) environment in the fasted state taken from Paraiso and co-workers (2019) (Paraiso et al., 2019). This figure shows that there is no difference in the rate and extent of dissolution between the simulated gastric and small intestine environments.

After developing and validating the PBPK/PD model to represent the fed state after a low fat meal, it was extrapolated to the fasted state. Fig. 8 depicts the simulated plasma profile together with the in vivo data obtained from Weinling and co-workers (2006) (Weinling et al., 2006b). The PD profile could not be verified externally because no in vivo data for $\%$ change in $\beta$-EEG under fasted state conditions is available in the open literature.

As previously mentioned, zolpidem belongs to BCS class I. Its solubility is not affected by $\mathrm{pH}$ within the range found in the stomach and upper small intestine (pH 1.2 to 6.8). In light of these characteristics, the clinical relevant dissolution specification for zolpidem was evaluated by simulating different dissolution rates at pH 6.8 (Fig. 9), ranging from $85 \%$ dissolved in $15 \mathrm{~min}$ to $85 \%$ dissolved in $120 \mathrm{~min}$. These simulated fasted state dissolution profiles were then entered as the small intestinal dissolution profile in the ADAM module. The results for the PK and PD responses of zolpidem using the Simcyp ${ }^{\circledR}$ model outputs are depicted in Fig. 10.

Fig. 10 shows the pharmacokinetic and pharmacodynamic parameters values obtained after inputting the simulated dissolution profile into the PBPK/PD model in fasted state for zolpidem. On the one hand the results suggest that different dissolution rates would affect the magnitude of $\mathrm{AUC}_{0-12 \mathrm{~h}}, \mathrm{AUCR}, \mathrm{C}_{\max }$, and $\mathrm{R}_{\max }$ values by less than $10 \%$ when $Q \geq 85 \%$ in 15,30 or $45 \mathrm{~min}$. On the other hand, when slowly 

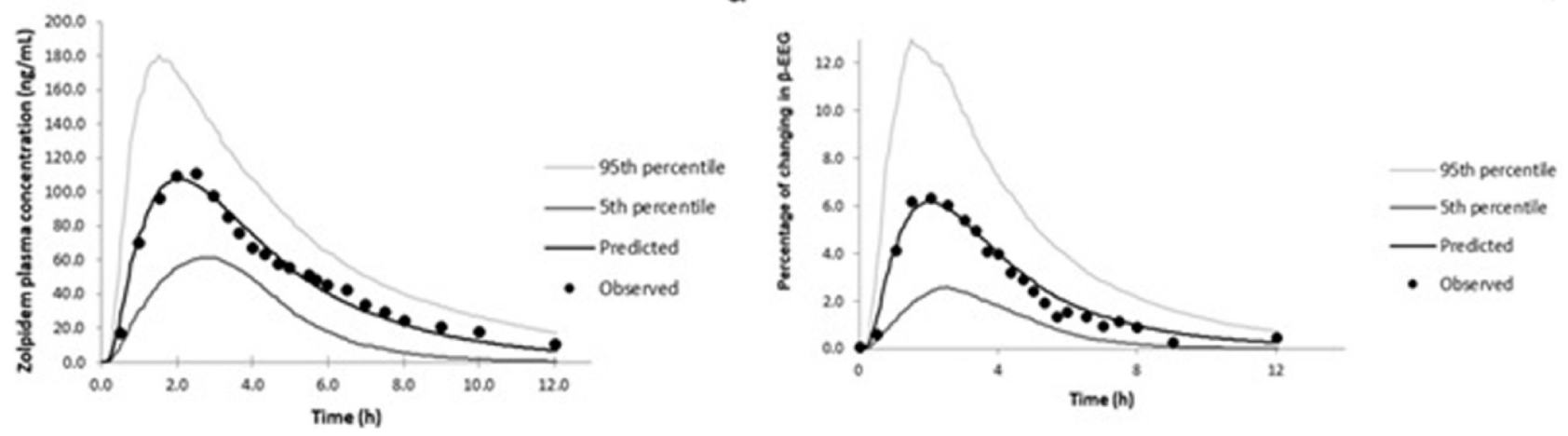

Fig. 4. (a) Mean observed ( $(\bullet)$ and model predicted PK profile (solid line) for health adults after administration of zolpidem IR tablets in the "low fed state". (b) Mean observed ( $\bullet$ ) and model predicted PD profile (solid line) after administration of zolpidem IR tablets over time.

Table 3

Pharmacokinetic parameters obtained in the clinical trial of zolpidem with the outcomes from simulations using in vitro data inputted from biorelevant dissolution tests simulating the fed state after a low fat meal.

\begin{tabular}{lllll}
\hline PK parameter & Simulated & Observed & \% difference & AFE/AAFE \\
\hline $\mathrm{C}_{\max }(\mathrm{ng} / \mathrm{mL})$ & 107.47 & 108.85 & -1.26 & - \\
$\mathrm{AUC}_{0-12}(\mathrm{ng} . \mathrm{h} / \mathrm{mL})$ & 577.23 & 565.78 & -1.98 & - \\
$\mathrm{T}_{\max }(\mathrm{h})$ & 2.00 & 2.02 & 0.02 & - \\
$\mathrm{AFE}$ & - & - & - & 1.07 \\
$\mathrm{AAFE}$ & - & - & - & 1.10 \\
\hline
\end{tabular}

Table 4

Zolpidem pharmacodynamic parameters obtained in the study with the corresponding simulation results using in vitro data input from biorelevant dissolution tests simulating the fed state after a low fat meal.

\begin{tabular}{lllll}
\hline PD parameter & Simulated & Observed & \% difference & AFE/AAFE \\
\hline $\mathrm{R}_{\max }$ & 6.22 & 6.35 & -2.05 & \\
$\mathrm{AUCR}_{0-12}(\mathrm{ng} . \mathrm{h} / \mathrm{mL})$ & 30.08 & 30.01 & -0.7 & \\
$\mathrm{TR}_{\max }(\mathrm{h})$ & 2.12 & 2.0 & 0.12 & \\
$\mathrm{AFE}$ & - & - & - & 1.11 \\
$\mathrm{AAFE}$ & - & - & - & 1.13 \\
\hline
\end{tabular}

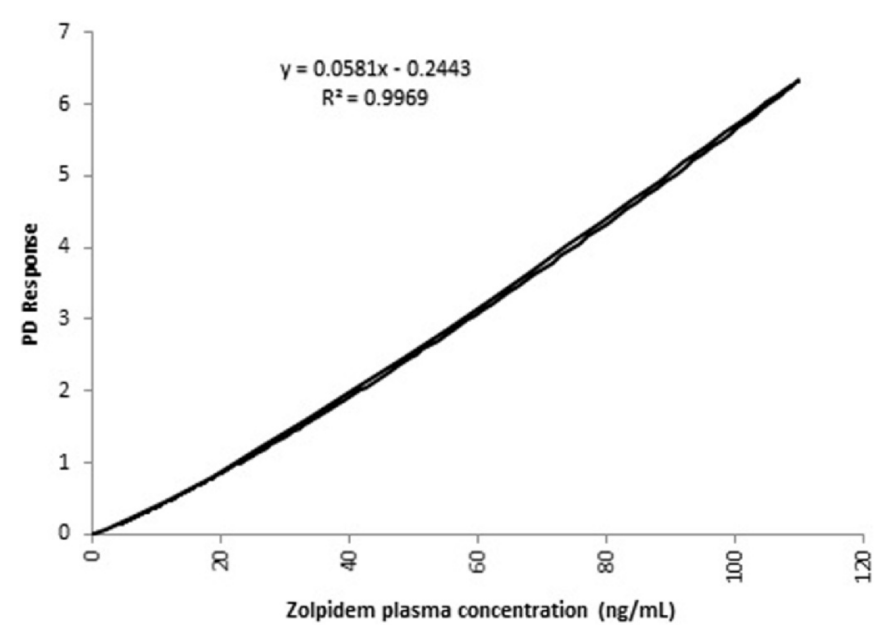

Fig. 5. Relationship between mean values of plasma concentration versus PD response of zolpidem.

dissolving tablets with $Q \geq 85 \%$ in 60 min were simulated, the $\mathrm{T}_{\max }$ and $\mathrm{TR}_{\max }$ were delayed to $1.7 \mathrm{~h}$, which translates into an expected delay in the onset of hypnotic effect compared with the response elicited by simulating the case of very rapidly dissolving tablets, $Q \geq 85 \%$ in 15 minutes. Therefore, the results suggest that only in the case of tablets with a slow dissolution of $Q \geq 85 \%$ in 60 minutes or more would lead to clinically significant changes in the time at which the maximum PK and PD responses are observed.

\subsection{Survey of clinical studies in the literature on the negative food effect for zolpidem immediate release tablets}

In the commercial label of Stilnox ${ }^{\circledast} 10 \mathrm{mg}$ IR tablets and in previous publications, zolpidem is described as having a negative food effect (Andreas et al., 2017; Greenblatt et al., 2013). Paraiso and coworkers (2019) published the mean plasma profile of the food effect investigation leading to this labeling and noted that the subject cohort consisted of 30 healthy male volunteers rather than a mixed-gender group (Paraiso et al., 2019).

Fig. 11a shows the mean plasma profile of zolpidem when administered in fasted and fed state. In that study, the coefficients of variation (CV) for $\mathrm{PK} \mathrm{T}_{\max }, \mathrm{C}_{\max }$ and AUC were tabulated, but variations at individual time points were not reported.

According to the mean profiles, lower values for both AUC and $\mathrm{C}_{\max }$ as well as a $60 \%$ prolongation in $\mathrm{T}_{\max }$ were observed in the fed compared to the fasted state for zolpidem IR. In both states, the results showed high CVs for all pharmacokinetic parameters: in the fasted state the CV for $\mathrm{C}_{\max } 42 \%$ and for AUC $56 \%$, while in the fed state, $\mathrm{C}_{\max }$ showed a $43 \%$ CV and AUC showed a $52 \% \mathrm{CV}$. The fed/fasted ratios for AUC and $\mathrm{C}_{\max }$ were 0.86 and 0.76, respectively, indicating that in the fed state, AUC and $\mathrm{C}_{\max }$ were decreased on the average by $\sim 15 \%$ and $25 \%$, respectively, compared to the fasted state, suggesting a negative food effect (Paraiso et al., 2019).

The PBPK model we developed for zolpidem IR also suggests that in the fed state $\mathrm{C}_{\max }$ and AUC would be somewhat decreased and the $\mathrm{T}_{\max }$ prolonged (Fig. 11b and c). Another important aspect is that the simulations captured the high inter-individual variability in the zolpidem plasma profile in both prandial states well.

To better understand the food effect, a literature search was conducted to locate publications of zolpidem IR clinical trials that had been conducted under fasted or fed conditions and compare their pharmacokinetic parameters $\left(\mathrm{C}_{\max }\right.$ and AUC). A total of 13 studies were located, 9 of which had been conducted in the fasted state and 4 in the fed state. In Fig. 12 the values of $\mathrm{C}_{\max }$, AUC and $\mathrm{T}_{\max }$ for these trials are depicted.

Studies listed in the Figure 12

Weinling et al., 2006. Study in male healthy volunteers.

$2 \quad$ Stilnox $^{\circledast}$ - FDA label food effect study, 1992. Fasted state arm (Male healthy volunteers).

3 Zolpidem - Public Assessment Report Netherlands, 2010. Study with male healthy volunteers. 

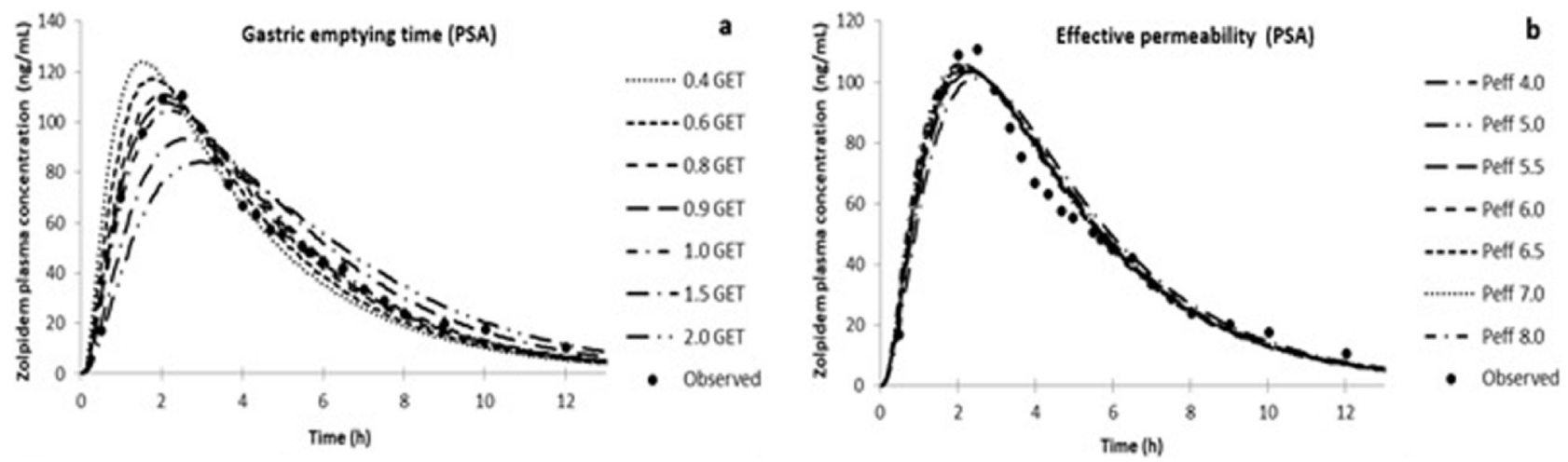

Fig. 6. Zolpidem pharmacokinetic profile applying PSA: (a) on GET and (b) on Peff in the fed state after a low fat meal.

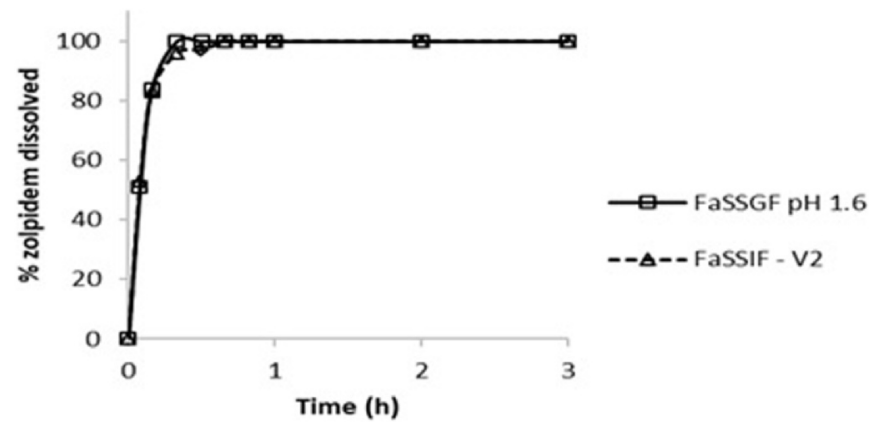

Fig. 7. Mean dissolution profiles of $10 \mathrm{mg}$ immediate release Stilnox ${ }^{\circledast}$ tablets in biorelevant Level II dissolution media simulating the fasted state using USP apparatus II. Error bars lie within the symbols in all cases. Reproduced with permission from Paraiso et. al (2019).

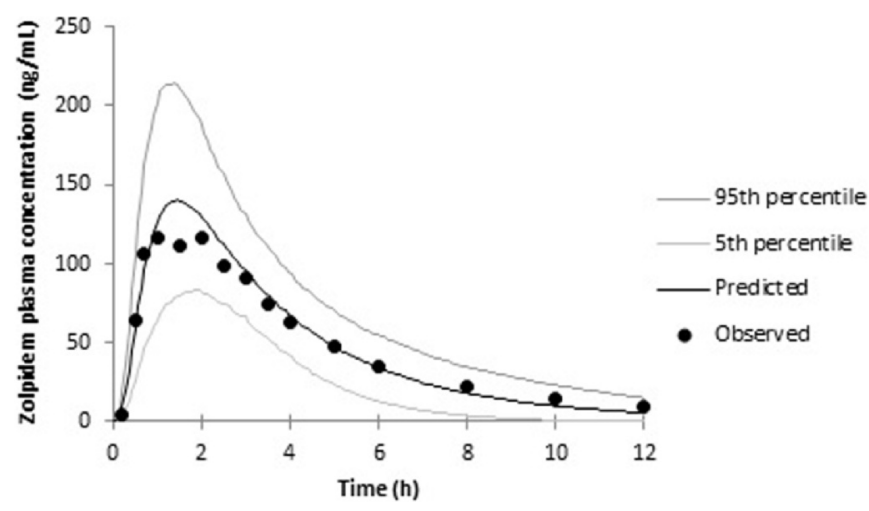

Fig. 8. Simulated and observed mean plasma zolpidem concentrations after simulation of zolpidem administration in the fasted state.

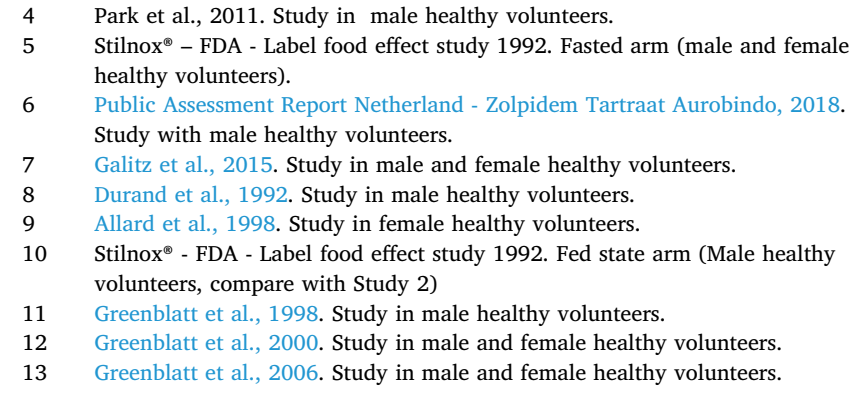

In these studies, the average (mean) values for $\mathrm{C}_{\max }$ and AUC in the fed state tended to be lower than in the fasted state, while the $\mathrm{T}_{\max }$ was mostly prolonged in the fed state compared to the fasted state.
However, there is some overlap in the pharmacokinetic parameters between the fed and fasted states.

\section{Discussion}

\subsection{Clinically relevant dissolution specifications for zolpidem IR products}

To arrive at clinically relevant dissolution specifications, it is important to establish a relationship between in vitro experiments and in vivo studies. Although there is large variability in the gastrointestinal physiology among volunteers/patients and thus challenges in establishing relationships between in vitro and in vivo data, biorelevant dissolution and PBPK modeling platforms provide an important way forward to establishing such relationships (Hermans et al., 2017; Markopoulos et al., 2015; Tsume et al., 2018). Drugs belonging to BCS class I and III in products with fast/very rapid dissolution are considered low risk products, for which there is generally no need to establish an in vitro/in vivo correlation (IVIVC) (Hermans et al., 2017; Polli et al., 2008; Tsume et al., 2018). Although zolpidem is a BCS class I, it has a short half-life, which most probably contributes to its classification as a highly variable drug when given orally. Further, for hypnotic drugs like zolpidem a high exposure and rapid effect for hypnotic drugs is usually desired. Therefore, for zolpidem in particular, it is important to identify an in vitro/in vivo relationship in order to set clinically relevant dissolution specifications.

Although standard IVIVC approaches are often used to set the CRDS, zolpidem IR formulations are not amenable to classical Level A IVIVC. For this reason, we used the alternative approach consisting of PBPK modelling coupled with the PD response. This approach has the further advantage of linking the CRDS all the way to the PD response, whereas classical IVIVC stops at correlating the dissolution with the PK parameters. Dissolution media that can be used to set clinically relevant dissolution specification for drug products can be anywhere between Levels 0 and 3 in the classification according to Markopoulous and coworkers (2015) Level 0 represents the simplest composition, attempting to represent only the $\mathrm{pH}$ in a segment of the gastrointestinal tract. As the Level increases, the composition becomes more complex (Markopoulos et al., 2015). Since zolpidem belongs to BCS class I and its dissolution is not affected by the gastrointestinal $\mathrm{pH}$, theoretical dissolution curves at $\mathrm{pH} 6.8$ (Level 0 ) with different dissolution rates (Fig. 9) were implemented into the PBPK/PD model to generate the corresponding plasma profiles of this drug. As shown in the simulation results (Fig. 10), it appears that the PK and PD parameters would only be significantly impacted if the formulation has disintegration/dissolution issues corresponding to $\mathrm{Q} \geq 85 \%$ being reached after more than 45 minutes.

Further guidance from EMA, FDA and ICH describes the possibility of waiving in vivo studies for drugs that belong to BCS class I and III. With regard to dissolution performance, this biowaiver approach is 


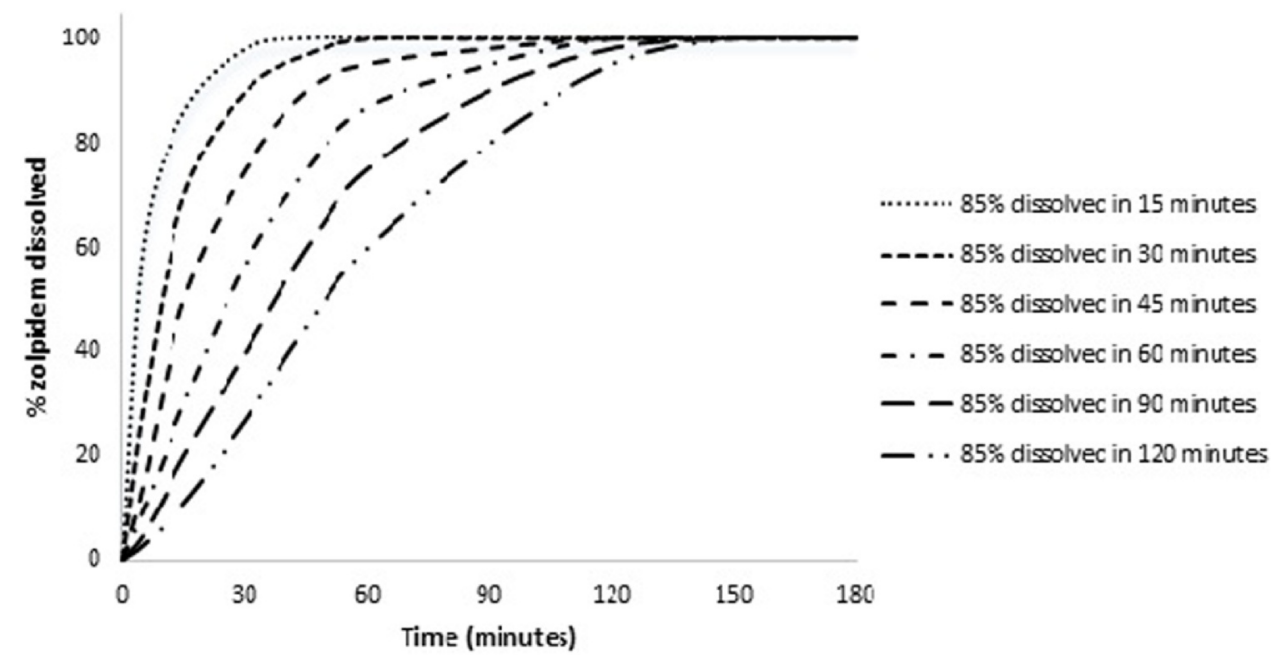

Fig. 9. Simulated dissolution profile in $\mathrm{pH} 6.8$ to determine the clinical relevant dissolution specification.

\section{PK parameters}
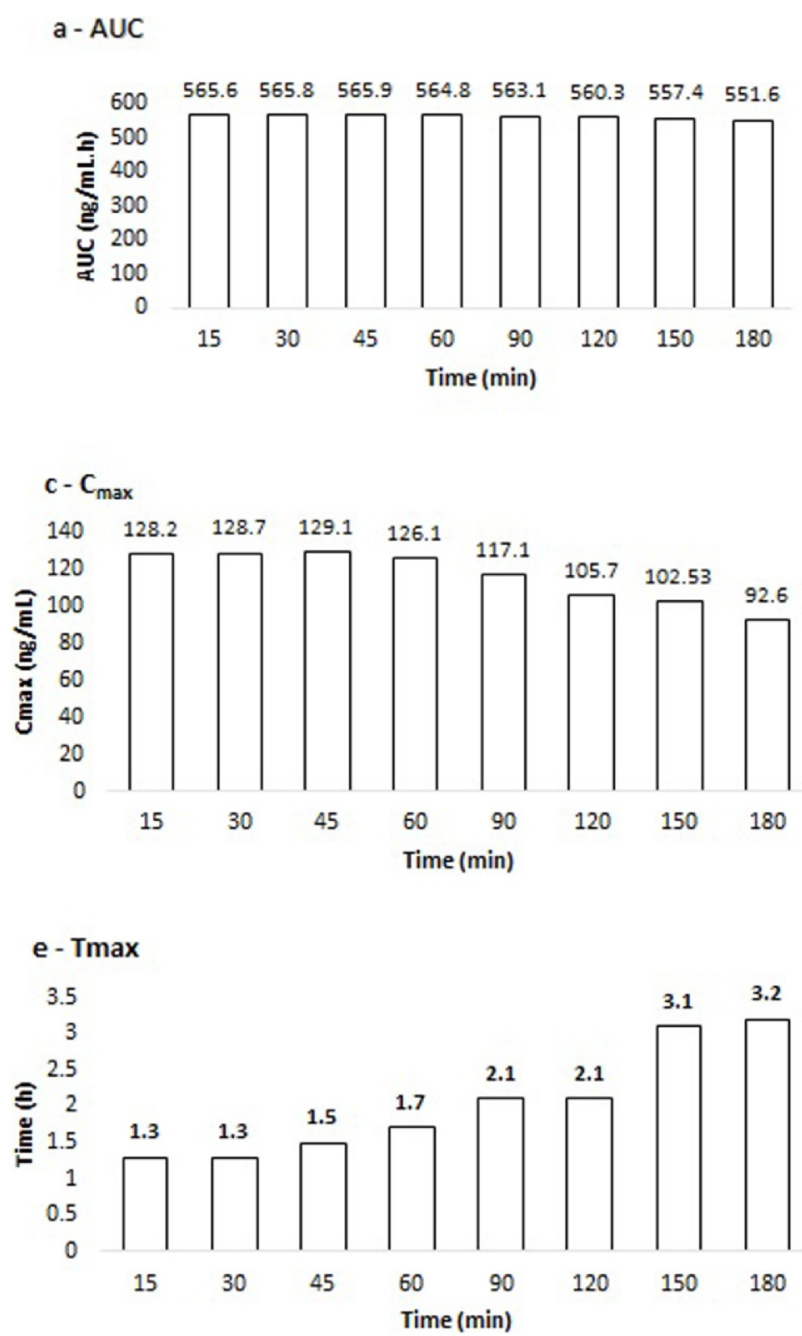

\section{PD parameters}
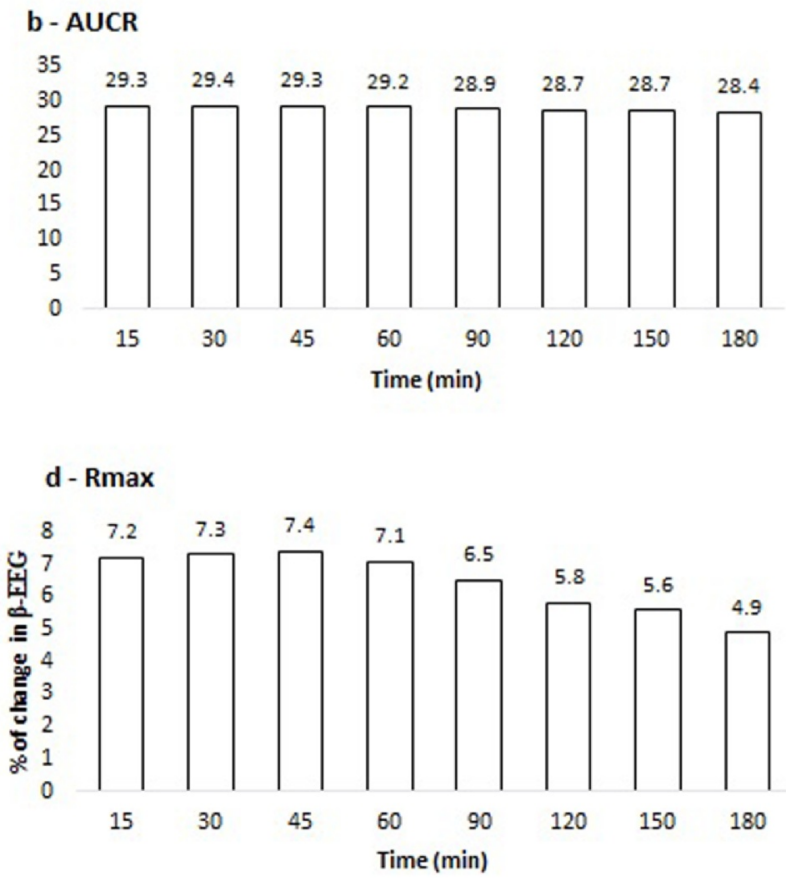

\section{f - TRmax}

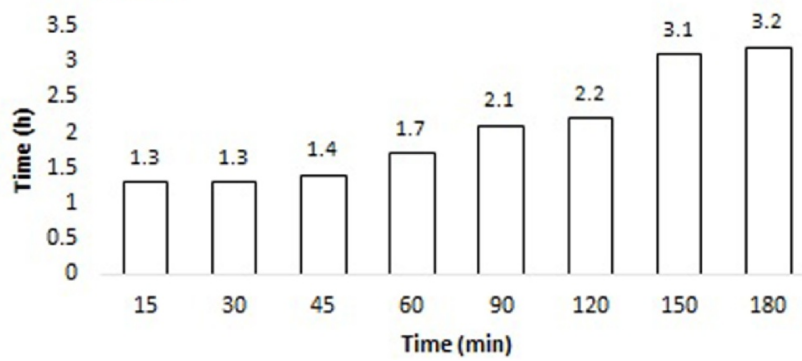

Fig. 10. Predicted dose-response after imputing the simulated dissolution profile into the PBPK/PD model of zolpidem. a, c and e represent the pharmacokinetic parameters and $\mathrm{b}, \mathrm{d}$ and $\mathrm{f}$ the pharmacodynamic parameters. 

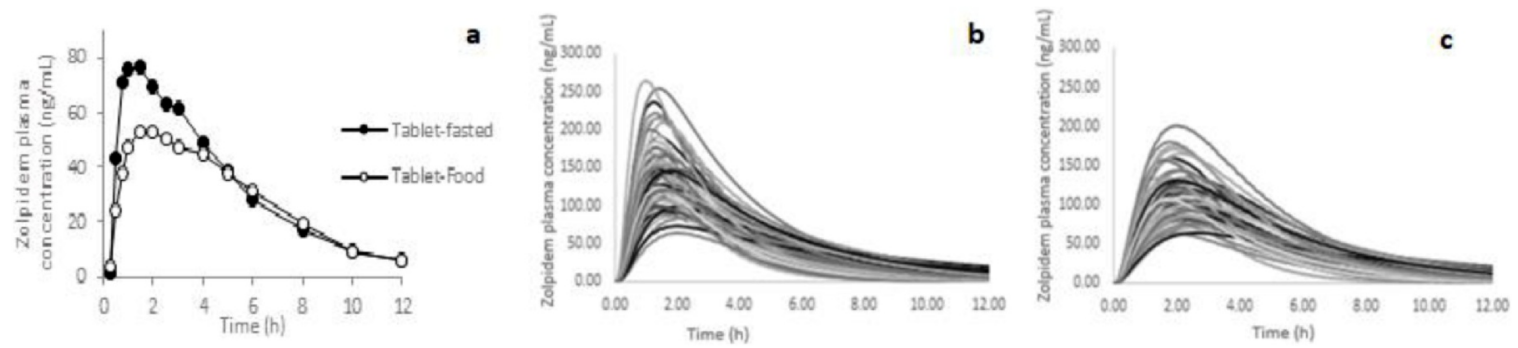

Fig. 11. a Mean plasma zolpidem concentration after oral administration of $10 \mathrm{mg}$ immediate release Stilnox ${ }^{\circledR}$ in 30 male subjects under fasting and fed conditions. (Reproduced with permission from Paraiso et al. (2019)). b and c. Simulated PK profiles in a virtual population of 70 healthy male and female volunteers after intake of $10 \mathrm{mg}$ zolpidem tablets. $\mathrm{b}$ fasted state and $\mathrm{c}$ fed state.

possible when the drug product dissolves very rapidly, which means that the tablets dissolve and release more than $85 \%$ of the labeled amount of the drug within 15 minutes, or when the drug formulation dissolves rapidly and releases $85 \%$ of the drug content within 30 minutes in compendial media at $\mathrm{pH} 1.2, \mathrm{pH} 4.5$, and $\mathrm{pH} 6.8$ (EMA, 2014; FDA, 2017; ICH, 2018). Comparing these limits to the PK/PD model for zolpidem IR tablets, suggests that the regulatory agencies could be somewhat more flexible with regard to the biowaiver criteria when zolpidem IR tablets are evaluated. According to the results obtained in our simulations, as long as $85 \%$ of the drug is released in 45 minutes or less, the impact on the PK and PD profiles of zolpidem would be minimal $(<10 \%)$.

Although the number of publications using dissolution data in conjunction with PBPK modeling and simulation has significantly increased in the last years, attempts to extend simulations through to clinical relevance by linking the results to a PD model have been few. Cristofoletti and Dressman (2014) used a PBPK/PD model to demonstrate that changes in pharmacokinetic parameters do not produce a proportional change in the antipyretic and anti-inflammatory activity of ibuprofen, such that even if two drug products are not bioequivalent with each other, they may still be therapeutically equivalent. This situation arises when the drug (in this case ibuprofen) has a flat dose- response relationship in the usual dosing range (Cristofoletti and Dressman, 2014). More recently, AbbVie used a PBPK modelling approach to broaden the dissolution criteria for Orilissa ${ }^{\circledR}$ (elagolix) tablets. According to the original specifications, two commercial batches failed to meet the dissolution acceptance criteria. But using the absorption modelling approach, the company was able to demonstrate to the FDA that even when the drug product releases the drug more slowly than the specifications require, there is no clinically significant effect on the exposure of the drug ("FDA Quality Asseessment for Elagolix Tablets," 2018). In a further example of using PBPK to set CRDS, Astra Zeneca proposed specifications for the particle size and dissolution rate of lesinurade from Zurampic ${ }^{\circledR}$ tablets that would ensure appropriate clinical performance of each batch, by running virtual bioequivalence trials in the Gastroplus PBPK platform (Pepin et al., 2016).

\subsection{Studies regarding the negative food effect of zolpidem}

Positive and negative food effects may be associated with the drug itself, the formulation and/or the physiology of the human gastrointestinal tract. Physiological parameters in the gastrointestinal tract e.g., gastric emptying time, release of bile salts and enzymes, gastrointestinal pH and splanchnic blood flow, are highly variable. Changes in

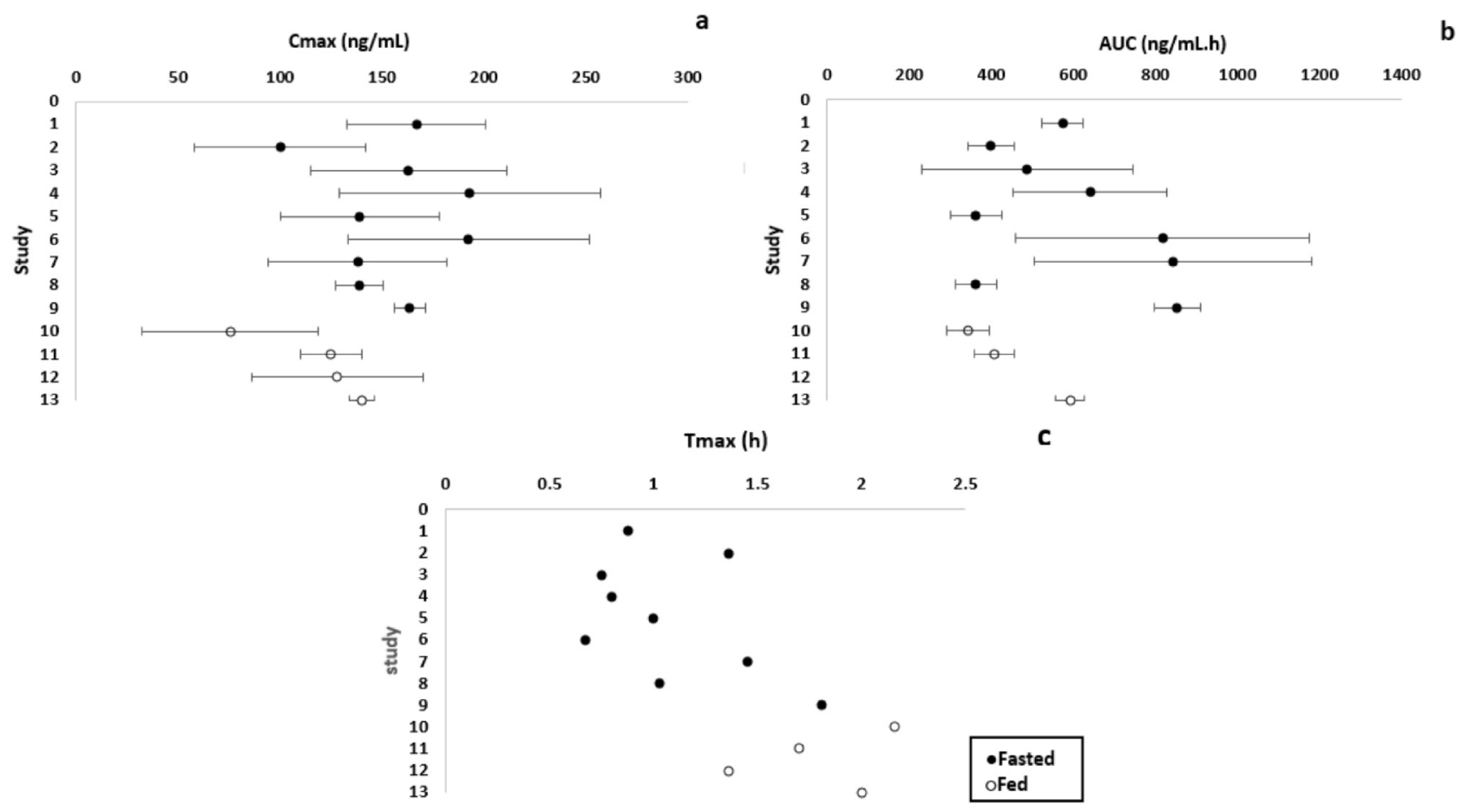

Fig. 12. Studies comparing the pharmacokinetic parameters of zolpidem when administered in the fasted and fed states. (a) Cmax, (b) AUC and (c) Tmax values. Closed dots ( represent studies in the fasted state and open dots $(O)$ in the fed state. In Fig. 13 (a) and (b) the values are presented as the mean and SD, in Fig. 13 (c) only by the average values. 
these parameters after a meal may alter the interaction the drug or dosage form with the gastrointestinal fluids. This complex combination of factors may lead to alterations in the luminal metabolism of drug, in vivo dissolution from the drug product, degradation of the drug and/or its absorption and thus a positive or negative food effect.

Attempts to describe the negative food effect observed for zolpidem with respect to AUC and $\mathrm{C}_{\max }$ showed that an increase in the fat content of the biorelevant media had little or no direct effect on the dissolution of zolpidem, however the longer gastric emptying time associated with a higher fat content could slow gastric emptying and thus indirectly affect the absorption profile (Paraiso et al., 2019). Similarly, when Andreas and co-workers (2017) tested the modified release formulation of zolpidem, they concluded that the negative food effect of zolpidem is associated with the delay in the gastric emptying time of the formulation. Additionally, it was shown that the release of zolpidem for the formulation can be influenced by high fat and/or protein content (Andreas et al., 2017).

In the zolpidem studies depicted in Fig. 12, the variability of $\mathrm{C}_{\max }$ and AUC was high in both the fasted and fed arms of the studies. These results are consistent with the fact that gastric emptying can vary widely among individuals, with the phase of the motility cycle in the fasted state, and between the fed and fasted states. Since zolpidem is highly soluble and permeable and has a short elimination half-life, the limiting step for absorption for this drug may well be the gastric emptying time (Andreas et al., 2017; Colo et al., 2015). For drugs with a short half-life, the $\mathrm{C}_{\max }$ becomes more sensitive to differences in the rate of gastric emptying and this sensitivity is exacerbated when solubility and permeability are not rate-limiting to absorption. Thus, the PK parameters of zolpidem, a BCS Class I substance, are particularly prone to intra and inter-individual variability in the gastric emptying time (Andreas et al., 2017; Markopoulos et al., 2015; Wagner et al., 2012). Although data from the literature combine to suggest that the zolpidem IR formulation has a negative food effect, the high variability in $\mathrm{T}_{\max }$, $\mathrm{C}_{\max }$ and AUC, the variability in the mean values from trial to trial and the paucity of trials in the fed state do not allow a firm conclusion to be reached. A larger crossover study in healthy men and women would be necessary to confirm the negative food effect indicated on the label.

\subsection{Administration of zolpidem in the fasted or fed state?}

The human circadian cycle can be different for each individual, therefore, the eating habits and bedtime can be specific for each person. Nevertheless, in general practice nutritionists and doctors instruct the patients to wait at least two to three hours between the last meal of the day and bedtime. This interval additionally allows for better digestion, preventing problems like heartburn at night and even insomnia (de Castro, 2004; De Zwaan et al., 2003; Schenck, 2006; Zwaan et al., 2006). Since zolpidem is used to sleep induction, it seems likely that most patients would take the product in the fed state. The marginal negative food effect could potentially decrease the effectiveness of the drug, while the delay in $\mathrm{T}_{\max }$ may incur a greater risk of residual sedation when compared with the fasting state. These considerations are in line with the product label, which indicates that for optimal effect Stilnox ${ }^{\circledast}$ tablets should not be taken with or immediately after a meal.

\section{Conclusions}

With the continuing evolution of PBPK models, physiologically based absorption modelling coupled with pharmacodynamics is emerging as an alternative methodology for development of in vitro-in vivo relationships. The integration of in vitro biorelevant dissolution with in silico methods such as PBPK or PBPK/PD is a promising tool to set clinically relevant dissolution specifications and thus link the lab to the patient. In this work, clinically relevant dissolution specifications for zolpidem immediate release products were set, showing that as long as drug release can meet a specification of $Q \geq 85 \%$ in 45 minutes or less, the product will have acceptable pharmacodynamic characteristics. This result suggests that the BCS-based biowaiver specifications ( $\mathrm{Q} \geq$ $85 \%$ in 30 minutes plus $\mathrm{f} 2>50$ or $\mathrm{Q} \geq 85 \%$ in 15 minutes) could be relaxed for zolpidem IR products and potentially for other BCS Class I drugs with short half-lives.

The high inter-subject variability in the PK parameters in the fasted and fed state suggest that for zolpidem IR products, absorption is largely determined by gastric emptying. The product label for zolpidem states that, due to delays in the gastric emptying and the associated lower $\mathrm{C}_{\max }$ and AUC, it is recommended that zolpidem IR tablets should be taken in the fasted state for faster onset of effect and higher exposure. However, a review of clinical studies from the open literature casts some doubt on the negative food effect for this drug and to settle this question, a crossover study in men and women should be conducted.

\section{Acknowledgements}

This work was supported by the European Union's Horizon 2020 Research and Innovation Programme under grant agreement No 674909 (PEARRL).

\section{Supplementary materials}

Supplementary material associated with this article can be found, in the online version, at doi:10.1016/j.ejps.2020.105534.

\section{References}

Allard, S., Sainati, S., Roth-Schechter, B., Macintyre, J., 1998. Minimal interaction between fluoxetine and multiple-dose zolpidem in healthy women. Drug Metab. Dispos. $26,617-622$.

Amidon, G.L., Lennernäs, H., Shah, V.P., Crison, J.R., 1995. A theoretical basis for a biopharmaceutic drug classification: the correlation of in vitro drug product dissolution and in vivo bioavailability. Pharm. Res. An Off. J. Am. Assoc. Pharm. Sci. 12, 413-420.

Andreas, C.J., Pepin, X., Markopoulos, C., Vertzoni, M., Reppas, C., Dressman, J.B., 2017 Mechanistic investigation of the negative food effect of modified release zolpidem. Eur. J. Pharm. Sci. 102, 284-298.

Colo, S., Gonzaíez-A, I., Mangas-Sanjuan, V., Gonzaíez-A, M., Pastoriza, P., MolinaMartínez, I., Bermejo, M., García-Arieta, A., 2015. Investigating the discriminatory power of bcs-biowaiver in vitro methodology to detect bioavailability differences between immediate release products containing a class i drug. Mol. Pharm. 12, 3167-3174.

Cristofoletti, R., Dressman, J.B., 2014. Use of physiologically based pharmacokinetic models coupled with pharmacodynamic models to assess the clinical relevance of current bioequivalence criteria for generic drug products containing ibuprofen. J. Pharm. Sci. 103, 3263-3275.

Cysneiros, R.M., Farkas, D., Harmatz, J.S., von Moltke, L.L., Greenblatt, D.J., 2007. Pharmacokinetic and pharmacodynamic interactions between zolpidem and caffeine. Clin Pharmacol Ther 82, 54-62.

Darwich, AS., Neuhoff, S., Jamei, M., Rostami-Hodjegan, A., 2010. Interplay of metabolism and transport in determining oral drug absorption and gut wall metabolism: a simulation assessment using the "Advanced Dissolution, Absorption, Metabolism (ADAM) model. Curr. Drug Metab. 11, 716-729.

de Castro, J.M., 2004. The Time of Day of Food Intake Influences Overall Intake in Humans. J. Nutr. 134, 104-111.

de Haas, S.L., Schoemaker, R.C., van Gerven, J.M.a, Hoever, P., Cohen, aF., Dingemanse, J., 2010. Pharmacokinetics, pharmacodynamics and the pharmacokinetic/ pharmacodynamic relationship of zolpidem in healthy subjects. J. Psychopharmacol. 24, 1619-1629.

De Zwaan, M., Burgard, M.A., Schenck, C.H., Mitchell, J.E., 2003. Night time eating: A review of the literature. Eur. Eat. Disord. Rev. 11, 7-24.

Drover, D., Lemmens, H., Naidu, S., Cevallos, W., Darwish, M., Stanski, D., 2000. Pharmacokinetics, pharmacodynamics, and relative pharmacokinetic/pharmacodynamic profiles of zaleplon and zolpidem. Clin. Ther. 22, 1443-1461.

Durand, A., Thénot, J.P., Bianchetti, G., Morselli, P.L., 1992. Comparative pharmacokinetic profile of two imidazopyridine drugs: Zolpidem and alpidem. Drug Metab. Rev. 24, 239-266.

Eller, M.G., Della-Coletta, A.A., 1990. Absence of effect of food on alprazolam absorption from sustained release tablets. Biopharm. Drug Dispos. 11, 31-37.

FDA, 2007. Highlights of prescribing information - Ambien ${ }^{\circledR}$ and Stilnox ${ }^{\circledR}$ (zolpidem tartrate) [WWW Document]. URL http://www.fda.gov/medwatch (accessed 8 . 17.20).

FDA, 2017. Waiver of in vivo bioavailability and bioequivalence studies for immediaterelease solid oral dosage forms based on a biopharmaceutics classification system guidance for industry. 
Fotaki, N., Vertzoni, M., 2010. biorelevant dissolution methods and their applications in in vitro-in vivo correlations for oral formulations. Open Drug Deliv. J. 4, 2-13.

Galitz, L.A., Jayawardena, S., Furey, S.A., 2015. Pharmacokinetic effects of simultaneous administration of single-dose gabapentin $500 \mathrm{mg}$ and zolpidem tartrate $10 \mathrm{mg}$ in healthy volunteers: a randomized, open-label. Crossover Trial. Drugs R D 15, 71-77.

Greenblatt, D.J., 2010. Pharmacokinetic Determinants of the Clinical Effects of Benzodiazepine Agonist Hypnotics BT - GABA and Sleep: Molecular, Functional and Clinical Aspects. In: Monti, J.M., Pandi-Perumal, S.R., Möhler, H. (Eds.), Gaba and Sleep. Springer, Basel, Basel, pp. 95-118.

Greenblatt, D.J., Allen, M.D., MacLaughlin, D.S., Harmatz, J.S., Shader, R.I., 1978. Diazepam absorption: Effect of antacids and food. Clin. Pharmacol. Ther. 24, 600-609.

Greenblatt, D.J., Harmatz, J.S., Singh, N.N., Roth, T., Harris, S.C., Kapil, R.P., 2013. Influence of food on pharmacokinetics of zolpidem from fast dissolving sublingual zolpidem tartrate tablets. J. Clin. Pharmacol. 53, 1194-1198.

Greenblatt, D.J., Harmatz, J.S., von Moltke, L.L., Ehrenberg, B.L., Harrel, L., Corbett, K., Counihan, M., Graf, J.a, Darwish, M., Mertzanis, P., Martin, P.T., Cevallos, W.H. Shader, R.I., 1998. Comparative kinetics and dynamics of zaleplon, zolpidem, and placebo. Clin. Pharmacol. Ther. 64, 553-561.

Greenblatt, D.J., Harmatz, von Moltke, J.S., Wright, L.L., Durol, C.E., a, L., Harrel-Joseph, L.M., Shader, R.I., 2000. Comparative kinetics and response to the benzodiazepine agonists triazolam and zolpidem: evaluation of sex-dependent differences. J. Pharmacol. Exp. Ther. 293, 435-443.

Greenblatt, D.J., Legangneux, E., Harmatz, J.S., Weinling, E., Freeman, J., Rice, K., Zammit, G.K., 2006. Dynamics and kinetics of a modified-release formulation of zolpidem: comparison with immediate-release standard zolpidem and placebo. J. Clin. Pharmacol. 46, 1469-1480.

Hermans, A., Abend, A.M., Kesisoglou, F., Flanagan, T., Cohen, M.J., Diaz, D.A., Mao, Y., Zhang, L., Webster, G.K., Lin, Y., Hahn, D.A., Coutant, C.A., Grady, H., 2017. Approaches for establishing clinically relevant dissolution specifications for immediate release solid oral dosage forms. AAPS J 19, 1537-1549.

\section{ICH, 2018. International Council for Harmonisation (ICH) Draft Guideline} Biopharmaceutics Classification System-Based Biowaivers M9.

Jamei, M., Turner, D., Yang, J., Neuhoff, S., Polak, S., Rostami-Hodjegan, A., Tucker, G., 2009. Population-based mechanistic prediction of oral drug absorption. AAPS J 11, 225-237.

Jantratid, E., De Maio, V., Ronda, E., Mattavelli, V., Vertzoni, M., Dressman, J.B., 2009. Application of biorelevant dissolution tests to the prediction of in vivo performance of diclofenac sodium from an oral modified-release pellet dosage form. Eur. J. Pharm. Sci. 37, 434-441.

Klein, S., 2010. The Use of Biorelevant Dissolution Media to Forecast the In Vivo Performance of a Drug. AAPS J 12, 397-406.

Locniskar, A., Greenblatt, D.J., Zinny, M.A., Harmatz, J.S., Shader, R.I., 1984. Absolute bioavailability and effect of food and antacid on diazepam absorption from a slowrelease preparation. J. Clin. Pharmacol. 24, 255-263.

Markopoulos, C., Andreas, C.J., Vertzoni, M., Dressman, J., Reppas, C., 2015. In-vitro simulation of luminal conditions for evaluation of performance of oral drug products: Choosing the appropriate test media. Eur. J. Pharm. Biopharm. 93, 173-182.

Ms, A.Y., Kumagai, Y., Fujita, T., Hasunuma, T., Yokota, S., Maeda, M., Otani, Y., Majima, M., 2007. Different effects of light food on pharmacokinetics and pharmacodynamics of three benzodiazepines, quazepam, nitrazepam and diazepam. J. Clin. Pharm. Ther. $32,31-39$.

Norman, J.L., Fixen, D.R., Saseen, J.J., Saba, L.M., Linnebur, S.A., 2017. Zolpidem prescribing practices before and after Food and Drug Administration required product labeling changes. SAGE Open Med 5, 1-5.
Obach, R.S., Baxter, J.G., Liston, T.E., Silber, B.M., Jones, B.C., MacIntyre, F., Rance, D.J., Wastall, P., 1997. The prediction of human pharmacokinetic parameters from preclinical and in vitro metabolism data. J. Pharmacol. Exp. Ther. 283, 46-58.

Paraiso, R.L.M., Watanabe, A., Andreas, C.J., Turner, D., Zane, P., Dressman, J., 2019. Invitro-in-silico investigation of the negative food effect of zolpidem when administered as immediate-release tablets. J. Pharm. Pharmacol. 71, 1663-1676.

Paul, A.D., Wang, W.L., Paul, W.S., Andy, I.T., John, P.S., Ghahramani, P., Tracey, H., Linda, B., Sheena, B., Ryan, C.G., Abrahamsson, B., 2008. Mini-review themed issue: bioequivalence, biopharmaceutics classification system, and beyond clinical relevance of dissolution testing in quality by design. AAPS J 10, 280-290.

Pepin, X.J.H., Flanagan, T.R., Holt, D.J., Eidelman, A., Treacy, D., Rowlings, C.E., 2016. Justification of drug product dissolution rate and drug substance particle size specifications based on absorption pbpk modeling for lesinurad immediate release tablets. Mol. Pharm. 13, 3256-3269.

Polli, J.E., Abrahamsson, B.S., Yu, L.X., 2008. In vitro studies are sometimes better than conventional human pharmacokinetic in vivo studies in assessing bioequivalence of immediate-release solid oral dosage forms. AAPS J 10, 289-299.

Poulin, P., Theil, F.-P., 2009. Development of a novel method for predicting human volume of distribution at steady-state of basic drugs and comparative assessment with existing methods. J. Pharm. Sci. 98, 4941-4961.

Public Assessment Report Scientific Discussion Zolpidem tartarat Aurobindo $5 \mathrm{mg}$ and 10 mg, filmcoated tablets (zolpidem tartrate)[WWW Document], 2018. URLhttps:// www.geneesmiddeleninformatiebank.nl/Pars/h121164.pdf(accessed 3.8.19).

Ramirez, E., Laosa, O., Guerra, P., Duque, B., Mosquera, B., Borobia, A.M., Lei, S.H., Carcas, A.J., Frias, J., 2010. Acceptability and characteristics of 124 human bioequivalence studies with active substances classified according to the Biopharmaceutic Classification System. Br. J. Clin. Pharmacol. 70, 694-702.

Salvà, P., Costa, J., 1995. Clinical pharmacokinetics and pharmacodynamics of zolpidem. Therapeutic implications. Clin. Pharmacokinet. 29, 142-153.

Schenck, C.H., 2006. A study of circadian eating and sleeping patterns in night eating syndrome (NES) points the way to future studies on NES and sleep-related eating disorder. Sleep Med 7, 653-656.

Tsume, Y., Patel, S., Fotaki, N., Bergström, C., Amidon, G.L., Brasseur, J.G., Mudie, D.M., Sun, D., Bermejo, M., Gao, P., Zhu, W., Sperry, D.C., Vertzoni, M., Parrott, N., Lionberger, R., Kambayashi, A., Hermans, A., Lu, X., Amidon, G.E., 2018. Meeting report in vivo predictive dissolution and simulation workshop report: facilitating the development of oral drug formulation and the prediction of oral bioperformance. AAPS J 20, 100-108.

Vermeeren, A., 2004. Residual effects of hypnotics: epidemiology and clinical implications. CNS Drugs 18, 297-328.

Wagner, C., Jantratid, E., Kesisoglou, F., Vertzoni, M., Reppas, C., Dressman, J.B., 2012 Predicting the oral absorption of a poorly soluble, poorly permeable weak base using biorelevant dissolution and transfer model tests coupled with a physiologically based pharmacokinetic model. Eur. J. Pharm. Biopharm. 82, 127-138.

Weinling, E., Mcdougall, S., Andre, F., Bianchetti, G., Dubruc, C., 2006a. Pharmacokinetic profile of a new modified release formulation of zolpidem designed to improve sleep maintenance. Fundam. Clin. Pharmacol. 20, 397-403.

Weinling, E., McDougall, S., Andre, F., Bianchetti, G., Dubruc, C., 2006b. Pharmacokinetic profile of a new modified release formulation of zolpidem designed to improve sleep maintenance. Fundam. Clin. Pharmacol. 20, 397-403.

Zolpidem - Public Assessment Report Netherlands[WWW Document], 2010. URLhttps:// www.geneesmiddeleninformatiebank.nl/Pars/h103728.pdf(accessed 3.8.19).

Zwaan, M.de, Roerig, D.B., Crosby, R.D., Karaz, S., Mitchell, J.E., 2006. Nighttime eating: a descriptive study. Int. J. Eat. Disord. 39, 224-232. 